\title{
The research on reclaimed rubber desulphurization system based on expert system
}

\author{
Tao $\mathrm{Xu}^{1, \mathrm{a}}$, Yu Feng ${ }^{1, \mathrm{~b}}$, Zhifeng Wang ${ }^{1, \mathrm{a}}$ and Zhihua $\mathrm{Hu}^{1, \mathrm{a}}$ \\ ${ }^{1}$ Shanghai Polytechnic University, China \\ axutao@sspu.edu.cn, bfengyu@sspu.edu.cn
}

\begin{abstract}
Keywords: Reclaimed rubber; Expert system; Desulfurization; Twin screw extruding.
\end{abstract}
Abstract. In the process of reclaimed rubber producing, the desulfurization is an important link of waste rubber recycling. It is directly affect the quality of the recycling reclaimed rubber. At present, there is no good desulfurization way for industrialization. Therefore, how to carry on the desulphurization in the process of industrialization has become a problem to be solved immediately. In this paper, a reclaimed rubber desulphurization technology based on the expert system is proposed. Through the real-time feedback loop and control algorithm of expert system, the system control parameters can be regulated adaptively, so that the desulphurization effects can be achieved, and the efficiency of the recycling process and the technical feasibility are enhanced. It provides a technical idea and method for the rubber recycling.

\section{Technical Background}

In order to meet the increasing material performance requirements, the development of rubber is towards to the direction of high strength, wear resistance, stability and ageing resistance. But it brings a problem that is the waste rubber can't realize the natural degradation after a long time. Worsely a lot of waste rubbers cause the black pollution and the treatment of this pollution is much more difficult than that of plastic pollution. Meanwhile it is a waste of precious rubber source. All over the world, every year there are tens of millions of tons of waste rubbers produced, there into, in recent years, the quantity of waste rubbers in our country is more than ten millions of tons per year. It is a huge number. How to efficiently solve the problem has caused wide attention around the world. Ever the mountain of waste rubber products is burned as fuel. Since 1910, scientists from all over the world began to research more effective regeneration treatment technology of waste rubber[1]. The so-called reclaimed rubber means that the waste rubbers can be vulcanized again after through crushing, heating, mechanical processing and other physical and chemical processes. In fact, the essence of which is under a series of chemical and physical actions such as heat, oxygen, regenerative agent and mechanical action, the vulcanized rubber network damages degradation, and makes the rubber changed from elastic state into plastic and sticky state, so that it can be vulcanized again. The manufacture of reclaimed rubber mainly includes three parts, such as crushing, desulphurization and refining. There into crushing and refining belong to the mechanical processing, and desulfurization is a chemical treatment. In the manufacture of reclaimed rubber, the key working procedure is desulfurization. Under the action of mechanical, the rubber is processed into the powder particles. When the size of the powder particles is less than 1.5 millimeter, we call it as the vulcanized rubber powder. For the vulcanized rubber powder, there is no uniform classification method now. According to the different raw material of waste rubber, it can be divided into the tire rubber powder, rubber shoe powder, Sundry rubber powder and all kinds of special waste rubber powder. Reclaimed rubber as a filler occupies an important position in the rubber formulation system. With the adding of reclaimed rubber, the physical and mechanical properties of the raw rubber will be declined, such as tensile strength, modulus and tear strength. But some new characteristics that raw rubber does not have are derived. For example, the mixing time can be shortened, and the energy consumed during the rubber mixing process can be reduced. As well as, the feeding speed during the extrusion or extension process can be accelerated. When the mixing rubber contains reclaimed rubber, the issue of extrusion swelling and 
extension shrinking can be avoided. During the process of vulcanization, the reclaimed rubber has played a role in anti-aging properties, and the effect is better than adding other anti-aging agents.

As the manufacturing method of reclaimed rubber is different, the role characteristics, pressure and temperature during the desulphurization process of waste rubber are different, besides the nature and dosage of regenerative agent used is also different, moreover with different manufacturing methods of reclaimed rubber, the physical and chemical process caused the damage of rubber is different. Therefore, in the aspect of quality and yield, obvious differences are existed.

After the mechanical shear and pulverization, the waste rubber in the role of softener, activator and catalyst is processed by heating, oxygen treatments and machining, and it is converted into reclaimed rubber finally. Then the reclaimed rubber has the plastic property and can be re-crosslinking. At present, all above is the regeneration process of most reclaimed rubbers. The necessary factors during the regeneration process can be divided into four kinds. Respectively, they are mechanical action, heating, oxygen and regenerative agent. The matching of various factors is a complex process, so during the regeneration process, the matching between the best factors need to be selected and the balance of different process speeds need to be considered. In the process, various factors on the physical degradation and chemical degradation have influence on reclaimed rubber production process, mutual penetration and mutual restriction exist at the same time. In order to guarantee the producing effects of the reclaimed rubbers, most of the time experienced experts are needed to observe and guide the producing. For such a huge market of recycling waste rubber, the expert who can control the production technology of reclaimed rubber is relatively very lack, so how to use the knowledge of these experienced experts to service for the recycling waste rubber industry becomes a problem, which need to be solved immediately. So a control scheme of reclaimed rubber desulphurization system based on expert system architecture is put forward in this paper, further more, the duplication and digitization of the production experience can be realized.

\section{Expert System and Control Architecture}

Expert System. Expert system usually contains six parts, there are man-machine interface, knowledge base, reasoning machine, the interpreter, comprehensive database and knowledge acquisition. The Architecture of this system is shown as Fig. 1.There into the separation of knowledge base and reasoning machine makes it distinctive, and along with the variation of type, function and size, the architecture of expert system is different.

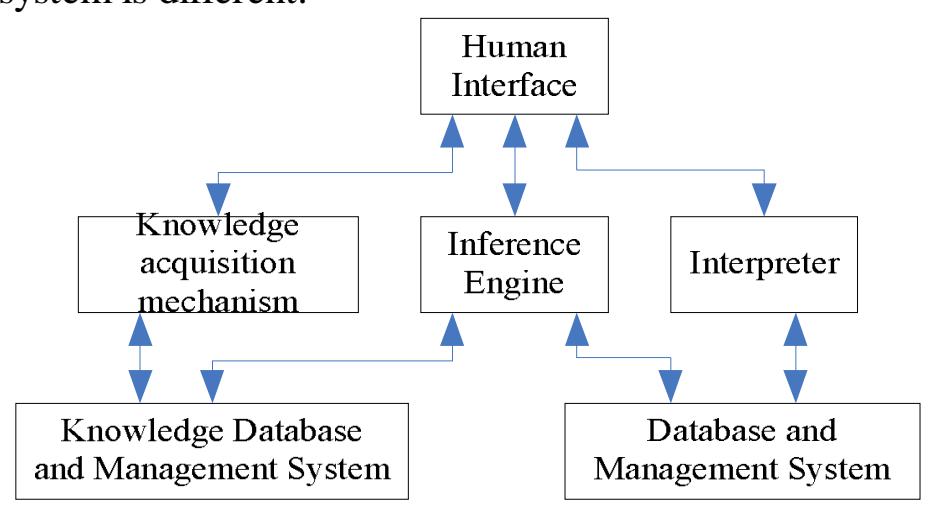

Fig. 1 Expert system architecture

In order to make the computer can use specialist areas knowledge, adopting a certain way to express knowledge is necessary. At present, the most basic method to realize the knowledge application is the way based on the rules of the production system. The production system mainly consists of comprehensive database, knowledge base and reasoning machine. Knowledge base is used to store the expert knowledge. Making use of the knowledge from the base, the expert system simulates the expert's thinking way to solve the issues, so the knowledge base is the key to determine whether the quality of expert system is superior, that is to say, the quality and the quantity of knowledge in the knowledge base determine the quality level of expert system. Generally speaking, the 
knowledge base and the expert system program are independent. Users can improve the performance of the expert system by changing and perfecting the knowledge in the knowledge base.

According to conditions or known information of the current problem, the reasoning machine matches the rules in the knowledge base repeatedly, then a new conclusion can be obtained and the resolving result of a problem can also be obtained. It seems like the thinking way of experts to solve problems. The knowledge base is through the reasoning machine to realize its value.

Comprehensive database is specially used to store the required raw data, intermediate result and final conclusion of reasoning process. It is usually used as a temporary storage area. According to the questions of the customers, the interpreter can make notes on the conclusion and solving process. It makes the expert system more human.

Knowledge acquisition is the key to determine whether the knowledge base of expert system is superior, it is also a "bottleneck" problem of expert system designing. Through the knowledge acquisition, the contents of the knowledge base can be expanded and modified, and the automatic learning function also can be realized[2-4].

For the expert system proposed in this paper, the programming language c\# is used, through the cooperation of artificial intelligence experts and reclaimed rubber experts, the expert knowledge base and the expert system are improved. Although the development cycle is relatively long and the degree of difficulty is great, it is flexible and practical. Furthermore, it can make the knowledge and experience of experts in this area be inherited effectively, and the manufacture of reclaimed rubber products be realized.

Mechanical Structure of Twin Screw Extruding Desulfurization System. Twin-screw extruding technology is a new method of recycling waste rubber. It combined the plastic processing with chemical crosslinking by using a continuous dynamic method. The waste tire rubber powder which is generated after pretreatment is processed by twin screw extruder[5-7]. Under the action of temperature, pressure, shear and vulcanizing agent, the waste rubber powder has quick and high degree of desulfurization and regeneration. But the current technology on the control mode is equivalent to the open loop system, namely the node temperature is detected by thermocouple, and compared with the threshold temperature. The structure of twin screw extruding desulfurization is shown as Fig.2. If it exceeds the threshold temperature, the separating module will stop heating, and begin cooling. Otherwise it will stop cooling and begin heating. The process is simple, but for the mixing status of vulcanizing agent, the vulcanizing effect and the mixing state of the twin-screw motor can't be monitored and handled, so it is difficult to produce stable performance products[8-9].

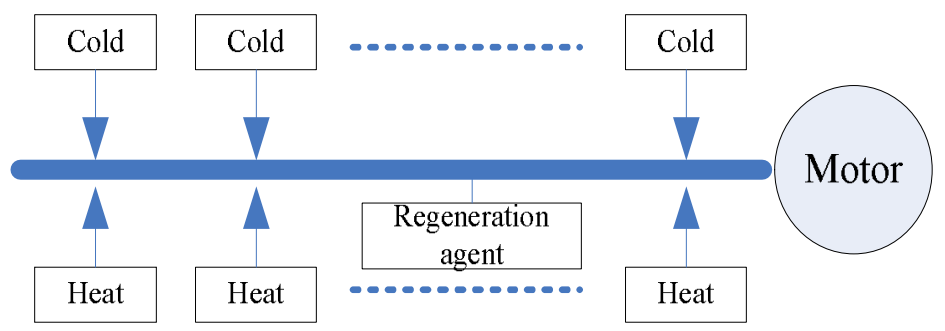

Fig. 2 The structure of twin screw extruding desulfurization

\section{Proposed Model and Solving Method}

Structure and Working Principle of the System Model. The whole solution architecture of the system put forward in this paper is shown in Fig. 3. 


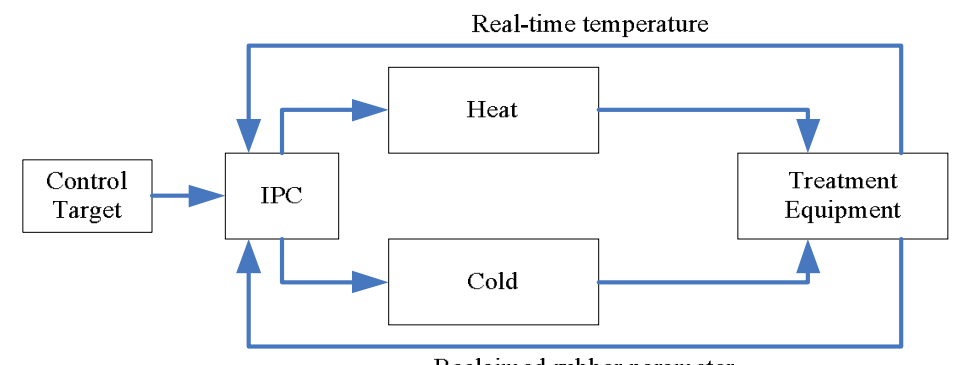

Fig. 3 The block diagram of Closed-loop control technology

For the above problems existing in the scheme of reclaimed rubber desulphurization, a control scheme based on closed loop system is proposed in this paper, and the closed loop control work is completed by the computer embedded in the expert system. For the system, Colloidal parameters, screw torque, node temperature and so on can be detected real time by sensors, then combined with the preset Colloidal target parameters, it can realize the automatic control of the system.

The control of twin screw colloidal heating uses the electromagnetic induction heater. The electromagnetic induction heater can sense the alternating current produced by heating power supply, and produce alternating magnetic field through the coils. The magnetic conductivity object is placed in the magnetic field and cuts the alternating magnetic field lines, so inside the object, the alternating current is produced. This makes the atom inside the objects move at a high speed without rule. The atoms collide and rub with each other, so the thermal energy is generated and it can play a role of heating. Namely it is a kind of heating way that converting electrical energy into magnetic energy, sensing magnetic energy and heating. With this way, the inefficient issue which can be caused by the way of heating transfer is resolved fundamentally.

For the cooling process, the flow of cooling water can be controlled with the method of proportional electromagnetic valve. It can make the flow change without interval, so that the temperature in the process of cooling can be ensured without mutation.

Intelligent design for real-time detection system of colloidal parameters is proposed in this paper, parameters such as the Mooney viscosity coefficient, hardness, density and so on can be measured and reflected to the expert system. The expert system with experience knowledge base compares the actual colloidal parameters with the preset target colloidal parameters, then it calls the experience knowledge judgment of the expert system and sets the parameters such as shear rate, node temperature and so on. According to the reasoning machine, the process is adjusted to be optimal automatically. Therefore reclaimed rubbers complying with the design requirements are produced[10].

Overall Physical Interaction Method.Overall system structure block diagram is shown in Fig. 4.

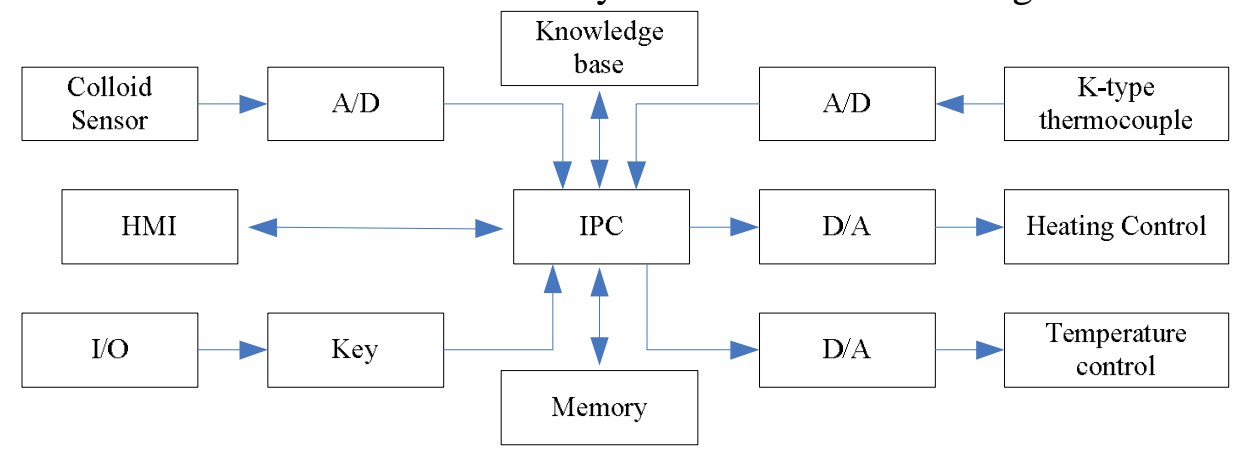

Fig. 4 System electrical wiring diagram

The sensor module including temperature detection and thermocouple detection module will transmit the detected data to the expert system. HMI mainly realizes the conversation between operator and control system, so as to realize the human-computer interaction functions with user friendly interface. The control of temperature signal is mainly through non-stop switching based on proportional solenoid valve, so that the temperature can switch rapidly and steadily. According to the preset rules and control scheme, the input process results and the tested data results, the expert 
knowledge base and reasoning machine will transfer the control parameters to the computer master control system to realize the control. The memory mainly storages the decision mechanism, strategy, control scheme and the optimal control strategy of different situations.

The overall system logic is shown as Fig. 5. The system logic is designed by the way of closed loop. After starting, the set target parameters are obtained first, according to the target parameters, the system craft is adjusted. During the working process, the colloidal parameters are detected continuously. Combined the experiential data of expert system with the reasoning machine, the system process is adjusted at real time, so that the quality parameters of the reclaimed rubber can achieve the system presupposed target parameters and requirements.

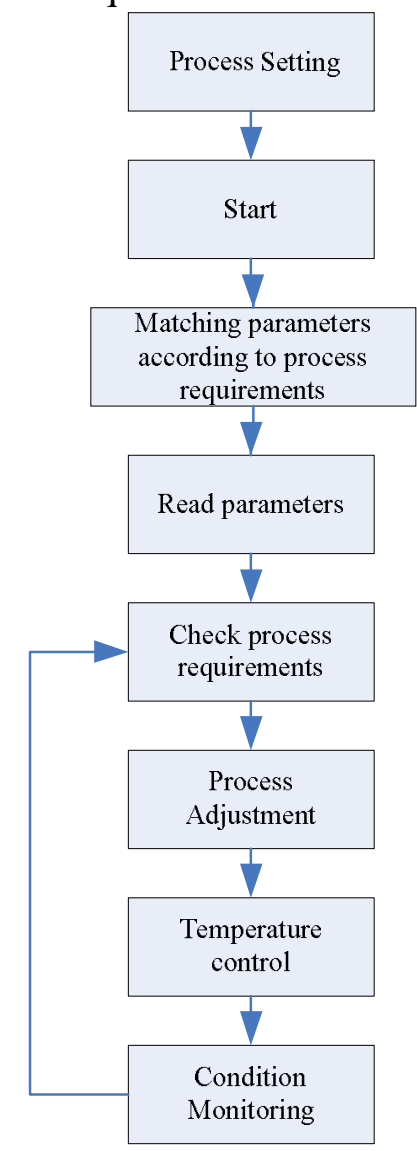

Fig. 5 The system control logic flow chart

\section{Conclusion}

In the design, the computer experience knowledge base, human-computer interaction module, and real-time colloidal detection module are all introduced into the treatment process of reclaimed rubber. It improved the system's practicability, convenience and adaptability. The introduction of experience knowledge base made the production line could produce different performance rubbers according to the user requirements at any time.

The core technology of this paper is that the experience Knowledge base was integrated into the heating treatment process after the addition of ingredients, and it improved the intelligence and efficiency of colloidal treatment process.

The monitoring device for detecting colloidal parameters in real time was introduced into the heating treatment process, so it allowed the real-time detection. It provided parameter basis for the intelligent self-learning and intelligent control.

The intelligent man-machine interface was introduced into the heating treatment process, so convenient interactive interface provided a powerful help to the system.

The relevant colloidal information was displayed directly on the human-computer interaction devices, it visually provided data support for the controller. 


\section{Acknowledgments}

This work was financially supported by the Shanghai Second Polytechnic University 2015 Young Teacher Training Program (B50YC150005-4) and Information and Communication Engineering (XXKZD1605).

\section{References}

[1] B.Adhikri, D.De, S.Maiti, Reclamation and recycling of waste rubber[J]. Progress in polymer science, 2000, 25(3):909-948.

[2] KUMAR K A, SINGH Y, SANYAL S. Hybrid approach using case-based reasoning and rule-based reasoning for domain independent clinical decision support in ICU[J]. Expert Systems with Applications, 2009, 36(1): 65 -71.

[3] Huang Jian, Hu Xiaoguang, Gong Yunan, et al. Machinery fault diagnosis expert system for high voltage circuit breaker[J]. Electric Machines and Control, 2011, 15(10): 43 -49.

[4] KO Y S. An expert system based on adaptive load transfer strategy for abnormal distribution system under heavy load condition[J]. Power Delivery, IEEE Transactions on, 2012, 27(3): 1162-1171.

[5] Sutanto P, Picchioni F, Janssen LPBM. EPDM rubber reclaim from devulcanized EPDM[J]. Applied Polymer Science, 2006, 102: 5028-5038.

[6] Bilgili E, Arastoopour H, Bernstein B. Inhomogeneous shearing deformation of a rubber-like slab within the context of finite thermoelasticity with entropic origin for the stress[J]. Non-Linear Mechanics, 2001, 36(6):887-900.

[7] Mouri M, Sato N, Okamoto H. New continuous recycling technology for vulcanized rubbers[J]. Polymer Recycling, 2000, 5(1): 37-42.

[8] Debapriya De, Amit Das, Debasish De. Effect of vulcaniztion technique on the physical properties of silica-filled EPDM rubber[J]. Applied Polymer Science. 2005, 99(3): 1132-1139.

[9] Otsuka S, Suzuki Y, Owaki M, et al. New recycling technology by shear flow stage control reaction for vulcanized rubber[C]. Austria:[s.n.], 1999: 255-261.

[10] Nagasaka Y, Nagashima A. Absolute measurement of the thermal conductivity of electrically conducting liquids by the transient hot-wire method[J]. Journal of Physics E: scientific Instruments: 1981: 14(12): 1435-1440. 